\title{
Epigenetic silencing of TGFBI confers resistance to trastuzumab in human breast cancer
}

Sònia Palomeras ${ }^{1 \dagger}$, Ángel Diaz-Lagares ${ }^{2,3 \dagger}$, Gemma Viñas ${ }^{1,4,5}$, Fernando Setien², Humberto J. Ferreira², Glòria Oliveras ${ }^{1,6}$, Ana B. Crujeiras ${ }^{7,8}$, Alejandro Hernández ${ }^{4}$, David H. Lum9 ${ }^{9}$ Alana L. Welm9, Manel Esteller $2,10,11,12,13^{*}$ and Teresa Puig ${ }^{1 *}$

\begin{abstract}
Background: Acquired resistance to trastuzumab is a major clinical problem in the treatment of HER2-positive (HER2+) breast cancer patients. The selection of trastuzumab-resistant patients is a great challenge of precision oncology. The aim of this study was to identify novel epigenetic biomarkers associated to trastuzumab resistance in HER2+ BC patients.

Methods: We performed a genome-wide DNA methylation (450K array) and a transcriptomic analysis (RNA-Seq) comparing trastuzumab-sensitive (SK) and trastuzumab-resistant (SKTR) HER2+ human breast cancer cell models. The methylation and expression levels of candidate genes were validated by bisulfite pyrosequencing and qRT-PCR, respectively. Functional assays were conducted in the SK and SKTR models by gene silencing and overexpression. Methylation analysis in 24 HER2+ human BC samples with complete response or non-response to trastuzumabbased treatment was conducted by bisulfite pyrosequencing.

Results: Epigenomic and transcriptomic analysis revealed the consistent hypermethylation and downregulation of $T G F B I, C X C L 2$, and SLC38A1 genes in association with trastuzumab resistance. The DNA methylation and expression levels of these genes were validated in both sensitive and resistant models analyzed. Of the genes, TGFB/ presented the highest hypermethylation-associated silencing both at the transcriptional and protein level. Ectopic expression of TGFBI in the SKTR model suggest an increased sensitivity to trastuzumab treatment. In primary tumors, TGFBI hypermethylation was significantly associated with trastuzumab resistance in HER2+ breast cancer patients.

Conclusions: Our results suggest for the first time an association between the epigenetic silencing of TGFBI by DNA methylation and trastuzumab resistance in HER2+ cell models. These results provide the basis for further clinical studies to validate the hypermethylation of TGFBI promoter as a biomarker of trastuzumab resistance in HER2+ breast cancer patients.
\end{abstract}

Keywords: HER2+ breast cancer, Trastuzumab resistance, DNA methylation, TGFBI

\footnotetext{
* Correspondence: mesteller@idibell.cat; teresa.puig@udg.edu

†Sònia Palomeras and Ángel Diaz-Lagares contributed equally to this work.

${ }^{2}$ Cancer Epigenetics and Biology Program (PEBC), Bellvitge Biomedical

Research Institute (IDIBELL), Hospitalet de Llobregat, Barcelona, Catalonia,

Spain

${ }^{1}$ New Therapeutics Targets Lab (TargetsLab), Department of Medical

Sciences, University of Girona, E-17071 Girona, Catalonia, Spain

Full list of author information is available at the end of the article
}

(c) The Author(s). 2019 Open Access This article is distributed under the terms of the Creative Commons Attribution 4.0 International License (http://creativecommons.org/licenses/by/4.0/), which permits unrestricted use, distribution, and reproduction in any medium, provided you give appropriate credit to the original author(s) and the source, provide a link to the Creative Commons license, and indicate if changes were made. The Creative Commons Public Domain Dedication waiver (http://creativecommons.org/publicdomain/zero/1.0/) applies to the data made available in this article, unless otherwise stated. 


\section{Background}

Breast cancer (BC) is the most common cancer in women worldwide and the leading cause of cancer deaths for women [1]. Approximately $15-20 \%$ of patients with this tumor overexpress the human epidermal growth factor receptor 2 (HER2) protein [2]. HER2 can activate downstream signaling cascades that induce cell proliferation through the Ras-mitogen-activated protein kinase (MAPK) pathway and inhibit cell death through the phosphatidylinositol 3'-kinase (PI3K)/protein kinase B (Akt)/ mammalian target of the rapamycin (mTOR) pathway [3, 4]. Characterizing HER2 as a proto-oncogene, a poor prognostic marker, and eventually as a therapeutic target has dramatically changed $\mathrm{BC}$ classification, risk assessment, and treatment [5]. The development of powerful targeted therapies directed specifically at HER2 has improved the survival of patients with both early-stage and metastatic BC.

Trastuzumab (Herceptin ${ }^{\oplus}$; Genentech, Inc., South San Francisco, CA, USA) is a humanized monoclonal antibody that selectively binds with high affinity to the extracellular domain of human HER2 protein and was the first targeted drug approved for treating HER2+ BC [6]. Although there are currently other anti-HER2 agents available, trastuzumab remains the gold standard for treating this subtype of BC. Despite the clinical benefits trastuzumab treatment in HER2+ BC brings, a large percentage of patients display primary or acquired resistance to the drug. In the last few years, several studies have focused on identifying the molecular mechanisms of trastuzumab resistance, such as aberrant activation of downstream signaling pathways [7] or the HER2 carboxyl-terminal fragments (CTF), also known as p95HER2, which are frequently found in HER2expressing BC cell lines and tumors [8]. Despite these efforts, the complete picture of the molecular mechanisms triggering trastuzumab resistance in $\mathrm{BC}$ remains unclear. Therefore, clarifying these mechanisms and identifying new resistance biomarkers is essential in the advance towards precision oncology in $\mathrm{BC}$ and the quest for new treatment options for those patients who do not respond to trastuzumab therapy.

DNA methylation is the most well-known epigenetic modification in humans and has been implicated in regulating the expression of a great variety of critical genes in cancer [9]. For this reason, DNA methylation status has emerged as one of the most promising epigenetic biomarkers for several types of cancer, including $\mathrm{BC}[10]$. These epigenetic markers can be useful in detecting tumors earlier or identifying patients with an increased risk of cancer, as well as evaluating disease progression or predicting the response to anticancer drugs [11]. In the last few years, some significant genes that are inactivated by promoter methylation in $\mathrm{BC}$ have been identified, including BRCA1 [12] and RASSF1A [13].
However, the analysis of hypermethylated genes in association with trastuzumab resistance is still a largely unexplored field that holds great potential.

The aim of this study was to evaluate the implication DNA methylation has in trastuzumab resistance and to identify epigenetically regulated genes with potential clinical value as biomarkers for trastuzumab resistance in HER2+ BC patients. To this purpose, we employed an integrative approach with genome-wide DNA methylation (450K array) and a transcriptomic analysis (RNASeq) in trastuzumab-sensitive and trastuzumab-resistant cell line models (SK and SKTR). In vitro results were validated in a cohort of 24 HER2+ tumor samples from patients experiencing both sensitivity and resistance to trastuzumab-based neoadjuvant therapy settings. Here, we determine epigenetic inactivation of the TGFBI gene by promoter $\mathrm{CpG}$ island hypermethylation, which are CpG-rich regions of DNA that are often associated with the transcription start sites of genes, with possible implications for trastuzumab-resistant BC pathways [14]. The hypermethylation of TGFBI also suggest its potential clinical usefulness as a biomarker for trastuzumab resistance in HER2+ BC patients.

\section{Methods \\ Cell culture}

SKBr3 (SK) and AU565 (AU) HER2+ breast carcinoma cells were obtained from Eucellbank (University of Barcelona, Spain) [15] and the American Type Culture Collection (ATCC, Rockville, MD, USA). SKBr3 and AU565 cells were routinely grown in McCoy's (Gibco) and Dulbecco's modified Eagle's medium (DMEM; Gibco), respectively, and supplemented with 10\% FBS (HyClone Laboratories), 1\% L-glutamine (Gibco), 1\% sodium pyruvate (Gibco), and $100 \mathrm{U} / \mathrm{mL}$ penicillin/streptomycin (HyClone Laboratories). Cell lines were kept at $37{ }^{\circ} \mathrm{C}$ and $5 \% \mathrm{CO}_{2}$ atmosphere. Long-term trastuzumab-resistant SK cells (SKTR) and AU565 cells (AUTR) had been previously developed in our laboratory $[16,17]$. Resistance was confirmed with cell viability assays. The trastuzumab-resistant SKTR and AUTR cells were maintained in $2 \mu \mathrm{M}$ of trastuzumab, i.e., a concentration in which parental cells were not viable.

\section{Patients and tissue samples}

TGFBI promoter methylation levels were retrospectively evaluated in tumor samples from 24 patients diagnosed with HER2+ BC at the Dr. Josep Trueta University Hospital, Girona (Spain) between 2007 and 2015. The patients were selected from the hospital's pharmacy database. The selection criterion included patients with early or locally advanced HER2+ BC who had received neoadjuvant treatment with trastuzumab and chemotherapy. Twenty patients had no response or partial response and 4 patients had complete response to trastuzumab plus chemotherapy. 
For all patients, hematoxylin and eosin (H\&E)-stained slides from formalin-fixed paraffin-embedded (FFPE) tumor blocks were examined to determine the representative areas of the invasive tumor. Estrogen receptor (ER), progesterone receptor (PR), and HER2 expression had been previously analyzed in the tumors using immunohistochemistry (IHC) . For each patient, clinical and histopathological features were obtained: age, stage (TNM classification [18]), histological grade (Bloom-Richardson grading system), menopause status, type of surgery, and relapse.

\section{5-Aza-2'-deoxycytidine treatment}

Epigenetic signatures are characterized by a very dynamic nature, where DNA methylation has often been shown as a reversible mechanism of transcriptional control by inhibition of enzymes such as the DNA methyltransferases [19]. We performed reactivation treatments using the demethylating agent, 5-aza-2'-deoxycytidine (5-aza-dC, Sigma-Aldrich, St Louis, MO) at $3 \mu \mathrm{M}$ and $5 \mu \mathrm{M}$ of 5 -aza-dC for $72 \mathrm{~h}$. The medium was changed every day to promote DNA demethylation.

\section{DNA and RNA isolation procedures}

Genomic DNA extraction from cell lines or formalin-fixed paraffin-embedded (FFPE) core biopsies $(10 \mu \mathrm{m})$ and tissue sections $(5 \mu \mathrm{m})$ using a QIAamp DNA Mini Kit and Deparaffinization Solution with a QIAamp DNA FFPE Tissue Kit (Qiagen, Hilden, Germany), respectively, were carried out following the manufacturer's instructions. For the RT-PCR experiments, cells were washed with PBS and then suspended in $1 \mathrm{~mL}$ of Qiazol (Qiagen Hilden, Germany) was added. Total RNA was isolated using a GeneJET RNA Purification Kit (Thermo Fisher Scientific) following the instructions provided by the manufacturer. All DNA and RNA samples were quantified using a NanoDrop 2000 Spectrophotometer (Thermo Fisher Scientific).

DNA was bisulfite-modified using an EZ DNA Methylation-Gold Kit (Zymo Research) in accordance with the manufacturer's recommendations.

\section{DNA methylation array}

Genome-wide DNA methylation analysis was performed using an Illumina $450 \mathrm{~K}$ DNA methylation microarray (Infinium HumanMethylation450 BeadChip) as previously described [20]. This technique is an epigenomic approach that allows analyzing the methylation profile of the human genome. Bisulfite-converted DNA from SK and SKTR models were used to hybridized on an Illumina Infinium HumanMethylation450 (450K) BeadChip array, following the Illumina Infinium HD Methylation protocol. Sex chromosomes were excluded from the analysis because they usually represent a high source of variation in DNA methylation levels [21]. Data was deposited into the NCBI Gene Omnibus, accession number: GSE123754.

\section{RNA sequencing}

DNA methylation is an epigenetic mechanism that usually affects $\mathrm{CpG}$ islands and promoters regulating the expression of the genes. For this reason, a transcriptomic analysis by RNA-Seq was performed. Library construction was performed using an Illumina TruSeq Stranded mRNA Sample Preparation Kit (CAT. No. RS-122-2101, RS-1222102). Following the transfer of the flow cell to an Illumina HiSeq instrument, a 101-cycle paired-end sequence run was performed using the HiSeq SBS Kit v4 sequencing reagents (FC-401-4002). A detailed description of the analysis is provided in the Additional file 1. Sequencing data have been posted in the Gene Expression Omnibus (GEO) database under accession number GSE114575.

\section{Bisulfite pyrosequencing}

To validate the results obtained from the arrays, bisulfite pyrosequencing analyses were performed. The primers for the PCR amplification and sequencing were designed with the PyroMark assay design software version 2.0.01.15. DNA-bs was amplified by PCR under standard conditions with biotinylated primers (see Additional file 1: Table S1) to convert the PCR product into singlestranded DNA templates. We used a Vacuum Prep Tool (Biotage, Sweden) to prepare single-stranded PCR products following the manufacturer's instructions. The PCR products were observed in $2 \%$ agarose gels before pyrosequencing. Reactions were performed in a PyroMark Q96 System version 2.0.6 (Qiagen, Hilden, Germany) using appropriate reagents and protocols. The methylation value was obtained from the average of the CpG dinucleotides included in the sequences analyzed using a Pyro Q-CpG 1.0.9 (Qiagen, Hilden, Germany).

\section{Methylation-specific PCR (MSP)}

DNA methylation was also validated using the methylationspecific PCR (MSP) using a set of primers designed by the Methyl Primer Express program for each gene analyzed. For each sample, two PCR reactions were produced using the same DNA template: one reaction with methylated primers (M: methylated) and the other with unmethylated primers (U: unmethylated). Commercial methylated human male genomic DNA (CpGenome Universal Methylated DNA, Millipore) was used as a methylated positive control (IVD), and DNA from normal lymphocytes (NL) as a positive control for unmethylated alleles. Primer sequences can be found in Additional file 1: Table S2.

\section{Gene expression analysis (qRT-PCR)}

To confirm the differential regulation of TGFBI, CXCL2 and SLC38A1 observed in SK and SKTR models used gold standard methodologies for gene expression such as quantitative real-time PCR. Total RNA was reversetranscribed into cDNA using a High Capacity cDNA 
Archive Kit (Applied Biosystems). Gene expression levels of selected genes were assessed using a LightCycler 480 Real-time PCR System (Roche) with a LightCycler 480 SYBR Green I Master (Roche). Primers are depicted in Additional file 1: Table S3. RT-PCR analyses were performed at least four times, and each gene was run in triplicate. GAPDH was used as an endogenous control to enable normalization.

\section{Short hairpin interference and ectopic expression assays} For the TGFBI long-term knockdown, two different ShRNAs were specifically designed against TGFBI mRNA (NM_000358) in two different loci, by considering a 19-base target sequence and inserted in the pLVX-shRNA2 vector (Clontech). For stable overexpression, the cDNA sequence of TGFBI was amplified from SKWT cDNA and cloned into the pLVX-IREStdTomato vector (Clontech). This construction (wild type) was then used as a template to create its mutated version following a PCR-based strategy. Mutations were performed in the RGD domain and the NKDIL (amino acids 354-358), YH18 (amino acids 563-580), and EPDIM (amino acids 617-621) motif in the second and fourth FAS-1 domains [22]. Lentiviral production was performed using the protocol described in Additional file 1. After lentiviral transduction, ZsGreen1-positive or tdTomatopositive cells were sorted by flow cytometry.

\section{Cell viability assays}

Cell viability was determined using a 3-(4, 5-dimethyl-2thiazolyl)-2, 5-diphenyl-2 h-tetrazolium bromide (MTT) assay. Briefly, cells were plated in their growth medium in 96-well plates at a cell density of $1.5 \times 10^{3}$ cells per well. After $24 \mathrm{~h}$, the growth medium was removed, and, $100 \mu \mathrm{L}$ of fresh medium containing the corresponding concentration of trastuzumab was added to each well for 5 days. Cell viability was measured using the standard colorimetric MTT assay as previously described [16]. Using the multi-well plate reader Benchmark Plus (BioRad), absorbance was determined to be $570 \mathrm{~nm}$. Data presented are from three separate wells per assay, and the assay was performed at least three times.

\section{Western blot analysis}

We previously demonstrated that different activation patterns of some HER receptors and their downstream signaling proteins are associated with the molecular mechanisms of acquired trastuzumab resistance (SK and SKTR) [16]. For this reason, a panel of the different proteins were analyzed in our models using Western blot analysis. The parental (SK and AU) and resistant (SKTR and AUTR) models were synchronized by starvation in serum-deprived medium $(0.5 \% \mathrm{FBS})$ for $24 \mathrm{~h}$.
Cells were lysed in ice-cold lysis buffer (Cell Signaling Technology, Inc.) with $100 \mu \mathrm{g} / \mathrm{mL}$ phenylmethylsulfonylfluoride (PMSF). Protein concentration was determined with Lowry (DC Protein Assay, Bio-Rad). Equal amounts of protein were heated in LDS Sample Buffer with Sample Reducing Agent (Invitrogen) for $10 \mathrm{~min}$ at $70{ }^{\circ} \mathrm{C}$, separated on SDS-PAGE and transferred to nitrocellulose membranes. Protein was detected using primary antibodies (Additional file 1: Table S5). Secondary antibodies, $\alpha$-tubulin and $\beta$-actin, conjugated to horseradish peroxidase were used. The immune complexes were detected using a chemiluminescent HRP substrate [SuperSignal $^{\mathrm{Tm}}$ West Femto Maximum Sensitivity Substrate (Thermo Scientific ${ }^{\mathrm{Tn}}$ Inc.) or Immobilon Western HRP Substrate (Millipore Sigma)] and in a Bio-Rad ChemiDoc ${ }^{\mathrm{Th}}$ MP Imaging System. Western blot analyses were repeated at least three times and representative results are shown.

\section{Statistical analysis}

Data were analyzed with Student's $t$ test when two groups were being compared or with one-way analysis of variance (ANOVA) followed by Tukey's honest significant difference (HSD) post hoc test or Tamhane's T2 post hoc test for multiple comparisons. The non-parametric KruskalWallis test was used when data did not follow normal distribution. When two groups were being compared, the data were analyzed with the Mann-Whitney $U$ tests for non-normally independent variables; otherwise, the Kruskal-Wallis test was used for more than two groups. In the patient cohort, we analyzed the TGFBI promoter methylation status and its association with the clinicalhistopathological characteristics. Patient data were summarized as median (first quartile-third quartile) for continuous variables, and frequencies and percentages for categorical variables. The potential association between clinical-histopathological characteristics and levels of TGFBI methylation (low $\leq 20 \%$, high $\geq 20 \%$ ) or differences in TGFBI methylation before and after treatment were analyzed using the Mann-Whitney $U$ and Kruskal-Wallis tests for continuous variables and the chi-square or Fisher exact tests, as appropriate, for categorical variables. The correlation between variables was observed using Spearman's rho coefficient. Characteristic (ROC) curves were used to assess the predictive capacity of the TGFBI marker. ROC curve analysis was performed using MedCalc software Version 18.11.3. The Hosmer-Lemeshow test and calibration plot was assessed with MedCalc and STATA software. Levels of significance were set at $p<$ 0.05 and are represented by asterisks, as follows: $p<0.05$ (denoted as *), $p<0.01$ (denoted as $* *$ ), and $p<0.001$ (denoted as $* * *)$. The statistical analysis was performed using the IBM SPSS software (Version 21.0; SPSS Inc.) 


\section{Results}

Genome-wide DNA methylation analysis in trastuzumabsensitive (SK) and trastuzumab-resistant (SKTR) breast cancer models

The Infinium HumanMethylation 450 BeadChip (450k array) is a validated tool for carrying out epigenomic projects because it allows the methylation status of approximately 450,000 CpGs located throughout the human genome to be detected [20]. Our group recently employed this approach in previous studies aimed at identifying genes regulated by DNA methylation for clinical applications such as biomarkers [23, 24]. Therefore, we took advantage of the $450 \mathrm{~K}$ array methodology to characterize the DNA methylation profile associated with trastuzumab resistance in $\mathrm{BC}$, and compared a trastuzumab-sensitive (SK) and trastuzumab-resistant (SKTR) BC models (Fig. 1a). The global analysis of the DNA methylation ( $\beta$ values) corresponding to the $\mathrm{CpG}$ sites with $p$ value $<0.01(469,927$ CpGs) showed differences in the scatter plot (Fig. 1b) between SK and SKTR models $\left(r^{2}=0.93\right)$ and revealed 27,314 differentially methylated CpGs $(\Delta \beta \geq 0.20)$ between both models. In particular, the number of $\mathrm{CpGs}$ that gained (red triangle in scatter plot) and lost (green triangle in scatter plot) a methylation level $\geq 0.20$ in SKTR with respect to SK was 14,845 and 12,469 , respectively.

Therefore, we focused our study on analyzing the methylation levels of the $\mathrm{CpG}$ sites located at the regulatory regions corresponding to $89,168 \mathrm{CpGs}$ and 13,508 genes. The supervised hierarchical clustering of the most variable CpGs from promoters and islands $(\Delta \beta \geq 0.20)$ showed a methylation pattern that clearly discriminated between the SK and SKTR models (Fig. 1c). Next, we used more stringent criteria to determine the most differentially methylated CpGs in the promoters and islands, considering in SKTR the CpGs with a methylation level $(\beta)$ in SK $<0.20$ and in SKTR $>0.60$ as hypermethylated, and in SKTR the CpGs with a methylation level $(\beta)$ in SKTR $<0.20$ and in SK $>$ 0.60 as hypomethylated. This analysis revealed 184 differentially methylated CpGs corresponding to 152 genes which, according to a Gene Ontology analysis (GO), were significantly associated (FDR < 0.05 ) with several biological processes related to cancer, such as cell adhesion pathways (GO:0007155), regulation of transcription (GO:0006355), development (GO:0007275), and control of apoptosis (GO: 0043065) (Fig. 1d). To verify whether the methylation observed in these genes had an impact on their transcriptional expression or not, we performed a transcriptomic analysis with RNA-Seq comparing the SK and SKTR models and obtained 1995 overexpressed and 3191 downregulated genes in SKTR displaying a fold change $\geq 1.5$ over the SK model. Then, we correlated the genes differentially methylated at the promoter and island with those differentially expressed between SK and SKTR (Fig. 1e). We identified 31 hypermethylated and downregulated genes and no gene hypomethylated and overexpressed in SKTR with respect to the SK model (Table 1). From this list of 31 genes, we selected three genes: TGFBI (transforming growth factor beta induced), CXCL2 (C-X-C motif chemokine ligand 2), and SLC38A1 (Solute carrier Family 38 Member 1) for further analysis. TGFBI, CXCL2, and SLC38A1 were selected because of the high number of differentially methylated CpGs ( $\geq 3 \mathrm{CpG}$ sites) they presented and their previous implications in $\mathrm{BC}$ [25-27].

\section{Epigenetic silencing of TGFBI, CXCL2, and SLC38A1 in trastuzumab-resistant cells (SKTR)}

First, we analyzed the DNA methylation of the selected genes by bisulfite pyrosequencing, which is a technique that allows single genes to be analyzed on a single CpG level [28]. We observed significantly higher methylation levels for the three genes in SKTR relative to the SK (Fig. 2a; $p<0.05$ for TGFBI and SLC38A1; $p<0.01$ for $C X C L 2)$. Like the previous transcriptomic analysis with RNA-Seq, the SKTR model also showed a significant reduction in the transcript expression levels of TGFBI, CXCL2, and SLC38A1 $(p<0.05)$ when compared to the SK cells analyzed by qRT-PCR (Fig. 2b). Interestingly, $T G F B I$ was the gene that showed the greatest decrease (fold change $=6.7$ ) in transcriptional levels in the SKTR model. Similar results were obtained by methylationspecific polymerase chain reaction (MSP) analysis (a very specific and sensitive method [29]) that showed higher methylation in SKTR with respect to the SK cells for all the genes evaluated (Fig. 2d). These results confirmed that hypermethylation of the three selected genes at the promoter and CpG island in SKTR cells is associated with reduce transcriptional levels.

In this sense, after treating the SKTR model with the demethylating agent 5-aza- 2 '-deoxycytidine (5-aza-dC) at $3 \mu \mathrm{M}$ and $5 \mu \mathrm{M}$ (Fig. 2c), the transcriptional levels of TGFBI, CXCL2, and SLC38A1 were significantly restored $(p<0.05)$, indicating that methylation is an epigenetic mechanism that has a functional role in the transcriptional control of the three genes in question. Importantly, this epigenetic silencing by DNA methylation observed was also confirmed at the protein level in two (TGFBI and CXCL2) of the three selected genes (Fig. 2e), showing a reduction of the protein levels in SKTR with respect to SK cells that was significantly restored in the hypermethylated SKTR model after the treatment with 5 -aza-dC $3 \mu \mathrm{M}$ and $5 \mu \mathrm{M}$. The change observed in the protein levels after the 5 -aza-dC treatment was especially drastic in TGFBI gene, where the absence of protein expression induced by the promoter hypermethylation in SKTR was completely recovered after the in vitro demethylation.

Taken together, these results suggest that TGFBI, an extracellular matrix (ECM) protein whose secretion is 
A

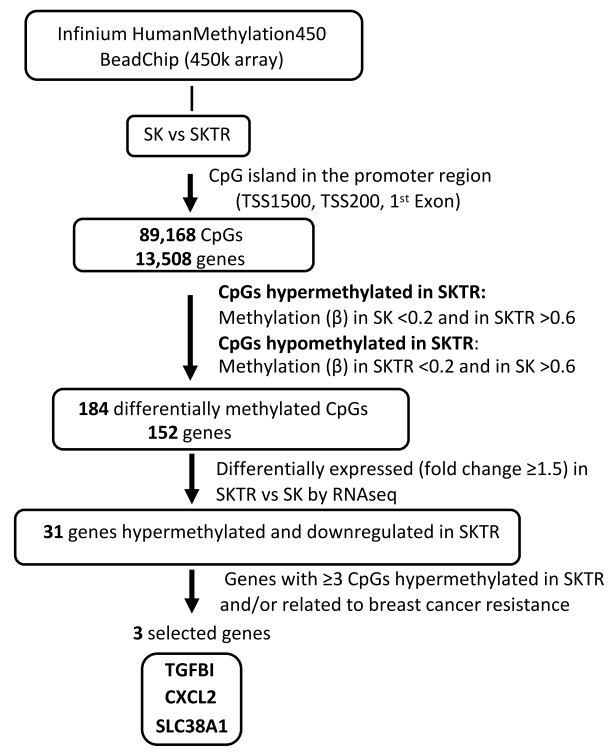

D

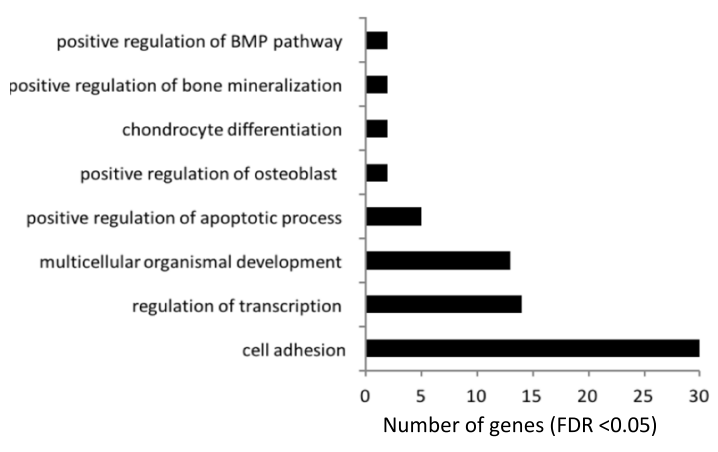

B

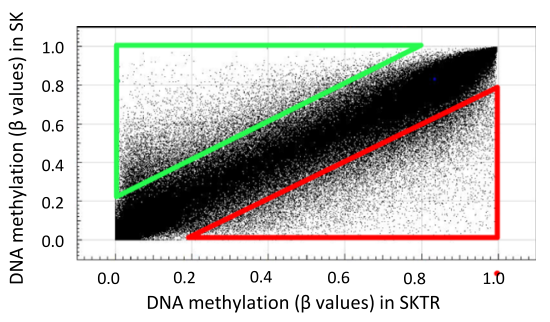

C

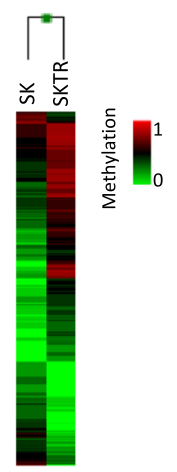

E
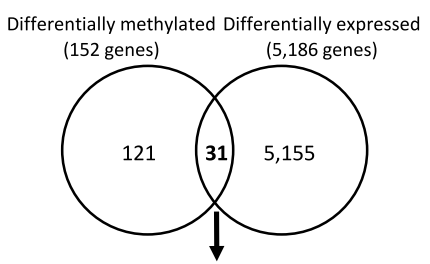

Hypermethylated and Downregulated in SKTR vs SK

$\begin{array}{llll}\text { ACTR3C } & \text { ANGPTL4 } & \text { B3GALNT1 } & \text { CXCL2 } \\ \text { CYP4X1 } & \text { ENTPD3 } & \text { FAHD2B } & \text { FAIM } \\ \text { HSF2BP } & \text { IGFBP7 } & \text { IL28RA } & \text { IRX6 } \\ \text { KCNJ12 } & \text { KIAA0895L } & \text { LOX } & \text { LRRC61 } \\ \text { LY6G5C } & \text { NR2F2 } & \text { PLIN5 } & \text { PTRF } \\ \text { RAB31 } & \text { RIN3 } & \text { RRP1B } & \text { SLC12A8 } \\ \text { SLC15A1 } & \text { SLC25A38 } & \text { SLC38A1 } & \text { SPDYA } \\ \text { TGFBI } & \text { WNT3 } & \text { ZNF532 } & \end{array}$

Fig. 1 Analysis of the DNA methylation profile associated to trastuzumab resistance in breast cancer cell lines. a Schematic flow chart used to identify differentially methylated genes associated to trastuzumab resistance after comparing the sensitive (SK) and resistant (SKTR) to trastuzumab human breast cancer cell lines. b Scatter plot representing DNA methylation normalized levels ( $\beta$ values) of SK and SKTR cell lines. Red and green triangles indicate the CpGs that gained and lost, respectively, a methylation level $\geq 0.20$ in SKTR with respect to SK cells. $\mathbf{c}$ Supervised hierarchical clustering of the most variable CpGs $(\Delta \beta \geq 0.20)$ from island and promoter regions between the SK and SKTR cell lines. $\mathbf{d}$ Summary of gene ontology (GO) analysis of the biological process categories for the 152 differentially methylated genes at CpG island and promoter levels between the SK and SKTR cell lines. e Venn diagram showing the differentially methylated and differentially expressed genes (obtained by RNA-Seq) between the SK and SKTR cell lines. The name of the 31 hypermethylated and downregulated genes in SKTR is indicated

induced by transforming growth factor- $\beta$ (TGF- $\beta$ ), is the most epigenetically regulated gene of the three selected.

Therefore, to verify that the results obtained for these genes were not specific to the SK and SKTR models, we extended their epigenetic analyses to other trastuzumabsensitive (AU) and trastuzumab-resistant (AUTR) human BC cell models (Fig. $2 \mathrm{f}$ and Additional file 2: Figure S1). Likewise, only TGFBI gene showed a high correlation between methylation, expression, and protein levels in the AUTR model. With MSP, we observed that hypermethylation of TGFBI in AUTR (compared to AU) was associated with a significant decrease $(p<0.05)$ in the transcriptional and protein levels of the gene. Importantly, the transcriptional and protein levels of TGFBI gene were both restored in the hypermethylated AUTR model after the treatment with 5 -aza-dC $3 \mu \mathrm{M}$ and $5 \mu \mathrm{M}$. These results confirm that the epigenetic silencing of TGFBI by DNA methylation is associated to trastuzumab resistance in human BC cells.

\section{The role of TGFBI expression in trastuzumab resistance cells (SKTR)}

We have shown that TGFBI promoter hypermethylation is highly associated with the downregulation of its 
Table 1 Thirty-one differentially methylated and differentially expressed genes between the SK and SKTR models

\begin{tabular}{|c|c|c|c|c|c|c|c|}
\hline TargetID & Chr & Position & Gene name & Gene region & SK & SKTR & SKTR vs SK \\
\hline cg08757148 & 1 & 24513722 & IL28RA;IL28RA;IL28RA & 1stExon;1stExon;1stExon & 0.11 & 0.67 & 0.56 \\
\hline cg03470088 & 1 & 24513939 & IL28RA;IL28RA;IL28RA & TSS200;TSS200;TSS200 & 0.05 & 0.79 & 0.75 \\
\hline cg26558485 & 1 & 47489282 & CYP4X1;CYP4X1 & 1stExon;5'UTR & 0.14 & 0.69 & 0.54 \\
\hline cg06816106 & 2 & 29033352 & SPDYA;SPDYA & TSS1500;TSS1500 & 0.18 & 0.64 & 0.46 \\
\hline cg14798656 & 2 & 97760745 & FAHD2B & TSS200 & 0.17 & 0.62 & 0.45 \\
\hline cg25999267 & 3 & 39424992 & SLC25A38;SLC25A38 & 1stExon;5'UTR & 0.19 & 0.75 & 0.56 \\
\hline $\operatorname{cg} 17264618$ & 3 & 40429014 & ENTPD3 & $5^{\prime} \cup T R$ & 0.14 & 0.61 & 0.46 \\
\hline cg09363539 & 3 & 124931746 & SLC12A8 & TSS200 & 0.01 & 0.82 & 0.81 \\
\hline cg15860013 & 3 & 138327718 & $\begin{array}{l}\text { FAIM;FAIM;FAIM;FAIM } \\
\text {;FAIM;FAIM;FAIM }\end{array}$ & $\begin{array}{l}\text { 5'UTR;TSS200;1stExon;5'UTR; } \\
\text { 1stExon;5'UTR;1 1stExon }\end{array}$ & 0.18 & 0.68 & 0.50 \\
\hline cg20841906 & 3 & 160822911 & $\begin{array}{l}\text { B3GALNT1;B3GALNT1; } \\
\text { B3GALNT1;B3GALNT1 } \\
\text {;B3GALNT1 }\end{array}$ & 5'UTR;5'UTR;5'UTR;5'UTR;TSS1500 & 0.06 & 0.70 & 0.64 \\
\hline cg20986370 & 4 & 57976171 & IGFBP7 & 1stExon & 0.19 & 0.69 & 0.49 \\
\hline cg19031658 & 4 & 74964856 & CXCL2;CXCL2 & 1stExon;5'UTR & 0.05 & 0.89 & 0.85 \\
\hline cg22847221 & 4 & 74964920 & CXCL2;CXCL2 & 1stExon;5'UTR & 0.13 & 0.61 & 0.48 \\
\hline cg00630212 & 4 & 74965135 & CXCL2 & TSS200 & 0.16 & 0.65 & 0.49 \\
\hline cg18804985 & 4 & 74965226 & CXCL2 & TSS1500 & 0.18 & 0.96 & 0.78 \\
\hline cg01429321 & 5 & 121413797 & LOX;LOX & 5'UTR;1stExon & 0.10 & 0.62 & 0.52 \\
\hline cg21034676 & 5 & 135364552 & TGFBI & TSS200 & 0.11 & 0.61 & 0.51 \\
\hline $\operatorname{cg} 14120129$ & 5 & 135364575 & TGFBI & TSS200 & 0.06 & 0.61 & 0.55 \\
\hline cg09873933 & 5 & 135364580 & TGFBI & TSS200 & 0.09 & 0.63 & 0.54 \\
\hline cg07151644 & 6 & 31649089 & LY6G5C & TSS1500 & 0.16 & 0.60 & 0.44 \\
\hline cg07753583 & 7 & 150020206 & LRRC61;ACTR3C;LRRC61 & TSS200;5'UTR;TSS200 & 0.17 & 0.86 & 0.69 \\
\hline $\operatorname{cg} 10348193$ & 7 & 150020240 & LRRC61;ACTR3C;LRRC61 & TSS200;5'UTR;TSS200 & 0.15 & 0.97 & 0.82 \\
\hline $\operatorname{cg} 11026333$ & 7 & 150020269 & LRRC61;ACTR3C;LRRC61 & TSS200;5'UTR;TSS200 & 0.06 & 0.98 & 0.92 \\
\hline cg01270001 & 7 & 150020401 & LRRC61;ACTR3C;LRRC61;LRRC61;LRRC61 & 1stExon;5'UTR;5'UTR;5'UTR;1 stExon & 0.13 & 0.94 & 0.81 \\
\hline cg22893248 & 7 & 150020751 & ACTR3C;ACTR3C;LRRC61;LRRC61 & 1stExon;5'UTR;5'UTR;5'UTR & 0.19 & 0.73 & 0.54 \\
\hline cg09327770 & 12 & 46663270 & SLC38A1;SLC38A1 & TSS200;TSS200 & 0.02 & 0.83 & 0.81 \\
\hline cg20463033 & 12 & 46663274 & SLC38A1;SLC38A1 & TSS200;TSS200 & 0.01 & 0.77 & 0.76 \\
\hline cg24795297 & 12 & 46663281 & SLC38A1;SLC38A1 & TSS200;TSS200 & 0.07 & 0.77 & 0.71 \\
\hline cg03859162 & 13 & 99404887 & SLC15A1;SLC15A1 & 1stExon;5'UTR & 0.12 & 0.66 & 0.55 \\
\hline cg03485262 & 14 & 92980031 & RIN3 & TSS200 & 0.04 & 0.72 & 0.68 \\
\hline cg08114373 & 14 & 92980204 & RIN3;RIN3 & 5'UTR;1 stExon & 0.17 & 0.61 & 0.44 \\
\hline cg18614734 & 15 & 96876248 & $\begin{array}{l}\text { NR2F2;NR2F2;NR2F2 } \\
\text {;MIR1469;NR2F2 }\end{array}$ & $\begin{array}{l}\text { Body;Body;5'UTR;TSS1500 } \\
\text {;TSS1500 }\end{array}$ & 0.18 & 0.65 & 0.47 \\
\hline cg01549404 & 16 & 55358636 & IRX6;IRX6 & 5'UTR;1stExon & 0.11 & 0.61 & 0.50 \\
\hline cg01568244 & 16 & 67218584 & KIAA0895L;EXOC3L & TSS1500;Body & 0.14 & 0.82 & 0.69 \\
\hline cg01666600 & 17 & 21279561 & KCNJ12 & TSS200 & 0.05 & 0.70 & 0.65 \\
\hline cg03928539 & 17 & 21279613 & KCNJ12 & TSS200 & 0.08 & 0.62 & 0.54 \\
\hline cg01637175 & 17 & 21281507 & KCNJ12 & $5^{\prime} U T R$ & 0.08 & 0.78 & 0.70 \\
\hline cg11804833 & 17 & 40575289 & PTRF;PTRF & 1stExon;5'UTR & 0.08 & 0.62 & 0.54 \\
\hline cg06093379 & 17 & 44896080 & WNT3;WNT3 & 5'UTR;1 stExon & 0.01 & 0.83 & 0.82 \\
\hline cg24441185 & 18 & 9708096 & RAB31 & TSS200 & 0.06 & 0.92 & 0.86 \\
\hline cg17289202 & 18 & 56530789 & ZNF532 & $5^{\prime}$ UTR & 0.13 & 0.80 & 0.67 \\
\hline
\end{tabular}


Table 1 Thirty-one differentially methylated and differentially expressed genes between the SK and SKTR models (Continued)

\begin{tabular}{|c|c|c|c|c|c|c|c|}
\hline TargetID & Chr & Position & Gene name & Gene region & SK & SKTR & SKTR vs SK \\
\hline cg22932336 & 19 & 4535070 & PLIN5 & $5^{\prime} \cup T R$ & 0.10 & 0.88 & 0.78 \\
\hline cg04532834 & 19 & 4535188 & PLIN5;PLIN5 & 1stExon;5'UTR & 0.17 & 0.64 & 0.46 \\
\hline cg02505409 & 19 & 8429160 & $\begin{array}{l}\text { ANGPTL4;ANGPTL4 } \\
\text {;ANGPTL4;ANGPTL4 }\end{array}$ & 5'UTR;1 1stExon;5'UTR;1 1stExon & 0.16 & 0.79 & 0.63 \\
\hline cg06837791 & 19 & 8429491 & ANGPTL4;ANGPTL4 & 1stExon;1stExon & 0.17 & 0.61 & 0.44 \\
\hline $\operatorname{cg} 12831261$ & 21 & 45078437 & RRP1B;HSF2BP & TSS1500;5'UTR & 0.01 & 0.93 & 0.92 \\
\hline
\end{tabular}

*Italics denotes the three final genes selected for further analysis

transcript and protein levels in two trastuzumabresistant models when compared to the corresponding sensitive models. Therefore, we next examined the functional contribution of epigenetic inactivation of the TGFBI gene to trastuzumab resistance. We depleted the endogenous TGFBI gene expression in the SK model by stable transfection with two different short hairpin RNAs (shTGFBI A and shTGFBI B). We also rescued the TGFBI expression in the TGFBI-hypermethylated and the downregulated SKTR models by stable transfection with a plasmid encoding the full-length TGFBI cDNA (TGFBI).

TGFBI is an extracellular matrix (ECM) protein and plays a role in mediating cell adhesion to the ECM, cell proliferation, adhesion, migration, and differentiation through interacting with collagen, fibronectin, laminin, and several integrins [30-32]. These integrin-binding properties of TGFBI have been related to different integrin-binding motifs located in fasciclin-1 domains, including NKDIL, YH18, and EPDIM as well as an ArgGly-Asp (RGD) domain [22]. For this reason, we became interested in determining whether this interaction between TGFBI with the ECM and integrins could be involved in the trastuzumab resistance in our models. With this objective in mind, we also expressed a mutated form of TGFBI with four different altered integrin binding motifs (NKDIL, YH18, EPDIM, and RGD), affecting cellular adhesion through the integrin interactions [22]. The transfection and validation protocols were the same as for the overexpression vector.

Despite its known function in cell adhesion and integrin-mediated signaling, we did not observe morphological changes after depletion, overexpression, or mutagenesis of TGFBI (Fig. 3a). The efficiency of the transfection was assessed by measuring the TGFBI gene expression using quantitative RT-PCR and Western blotting (Fig. 3b). A significant change in its levels was detected both after depletion and overexpression. Upon TGFBI transfection, we analyzed the resistance or sensitivity to trastuzumab in comparison to the parental and empty vector-transfected cells through an MTT assay over 5 days (Fig. 3c; Additional file 3: Figure S2). In the SK model, we observed that TGFBI depletion did not affect the trastuzumab response (Fig. 3c, upper panel). In contrast, overexpression of TGFBI in SKTR led to a significantly higher sensitivity at a trastuzumab concentration of $10 \mu \mathrm{M}$ compared to the resistant empty vector cell line (Mock: $p=0.020)$ and the SKTR model $(p=$ 0.004) (Fig. 3c, middle panel). The TGFBI mutated form (TGFBImut) showed the same response to trastuzumab treatment as the mock vector did, suggesting that TGFBI-mediated trastuzumab sensitivity requires the NKDIL, YH, EPDIM, and RGD binding motifs (Fig. 3c, lower panel).

Therefore, we examined HER family protein receptors and their downstream proteins related to PI3K/AKT and MAPK/ERK1/2 pathways for changes after TGFBI depletion, overexpression, and mutation (Fig. 3d). Consistent with the results of the trastuzumab cell viability, TGFBI depletion in SK cells showed no apparent changes in either total protein or activation levels for any of the proteins examined. Unlike TGFBI depletion, overexpression of TGFBI and TGFBImut in the SKTR model produced changes in the activation levels of some of the HER receptors and downstream signaling proteins in comparison with the mock control vector. In particular, overexpression of either wild type TGFBI or its mutated form resulted in a significant increase in the activation levels of HER1 (pHER1), HER2 (pHER2), and AKT (pAKT) compared to the SKTR model (Mock) that contains hypermethylated TGFBI without change in total levels of the respective proteins. Hence, TGFBI overexpression and its mutated form allow the trastuzumab-resistant model to adopt similar activation levels for HER1, HER2, and AKT as the trastuzumab-sensitive cells do.

These data suggest that selective overexpression of TGFBI in the SKTR model (hypermethylated for TGFBI) induces an increased sensitivity to trastuzumab and the activation of HER1 and HER2 receptors and the AKT downstream protein. Furthermore, the mutated domains from the second and fourth FAS- 1 regions of TGFBI are involved in its response to trastuzumab treatment, but not in its activation or interaction with HER2 downstream proteins. In summary, the SKTR model with an overexpression of TGFBI presents a behavior similar to the SK model. 
A

B
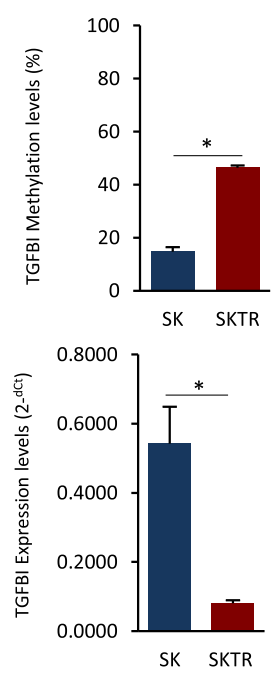

C

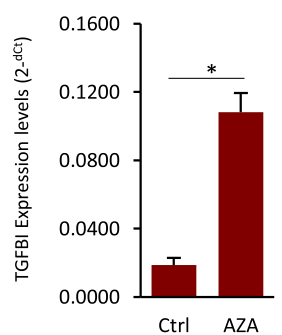

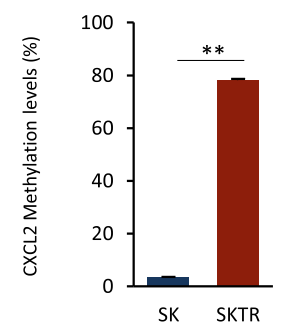
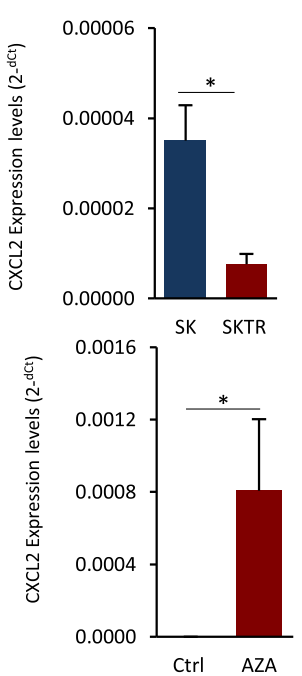
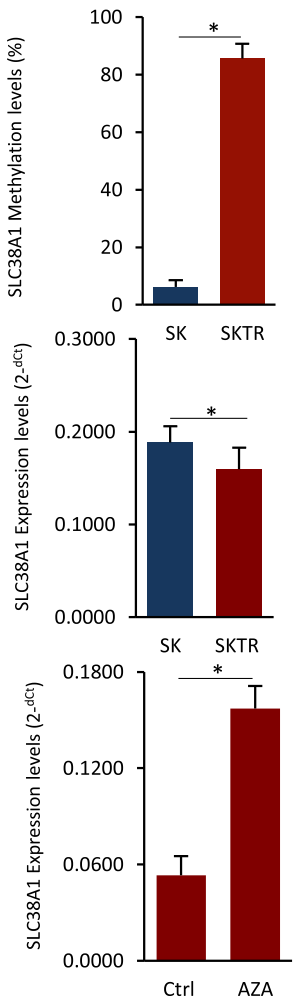

D

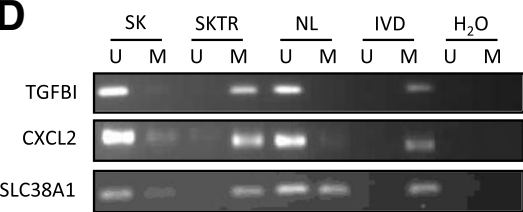

F

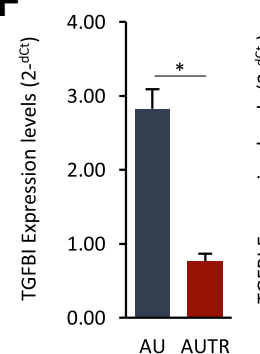

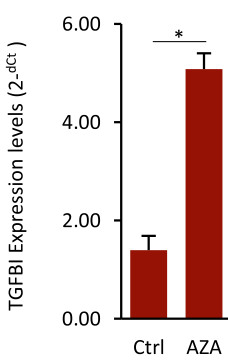

D

$$
\begin{aligned}
& \text { TGFBI } \\
& \text { CXCL2 } \\
& \text { SLC38A1 } \\
& \text { B-Actin }
\end{aligned}
$$
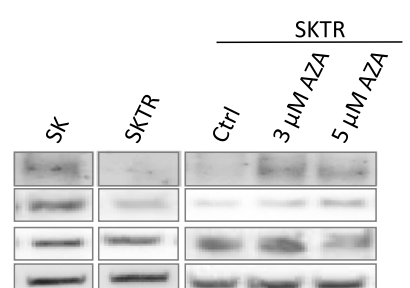

AUTR

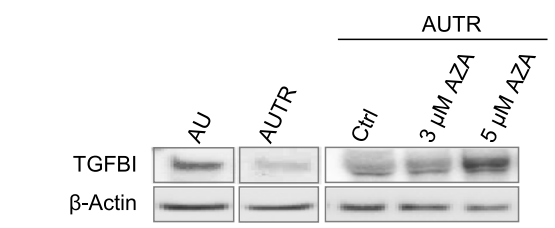

$\frac{A U}{U M} \frac{\text { AUTR }}{U M} \frac{N L}{U M} \frac{\text { IVD }}{U M} \frac{\mathrm{H}_{2} \mathrm{O}}{U M}$

TGFBI

Fig. 2 DNA methylation-associated silencing of selected genes comparing the trastuzumab-resistant and trastuzumab-sensitive cell line. a DNA methylation levels of TGFBI, CXCL2, and SLC38A1 in SK and SKTR cell lines by bisulfite pyrosequencing and $\mathbf{b}$ methylation-specific polymerase chain reaction (MSP) analysis. c Expression levels of TGFBI, CXCL2, and SLC38A7 in the unmethylated (SK) and methylated (SKTR) cell lines determined by qRT-PCR. $\mathbf{d}$ Restored expression of selected genes (TGFBI, CXCL2, and SLC38A1) after DNA demethylating agent 5-aza-2'deoxycytidine (5-aza-dC) in the SKTR methylated cell line by qRT-PCR. e Protein expression of all selected genes (TGFBI, CXCL2, and SLC38A1) in SK and SKTR cells before and after 5-aza-dC treatment by Western blot. f Analysis in AU and AUTR cell lines of TGFBI methylation by MSP (top) and (middle) its transcriptional (qRT-PCR) and (bottom) protein levels (Western blot) before and after 5-aza-dC treatment. In MSP, the presence of visible polymerase chain reaction products in lanes marked $U$ indicates unmethylated sequences; the presence of products in lanes marked $\mathrm{M}$ indicates methylated sequences. In vitro methylated DNA (IVD) was used as a positive control for methylated sequences. DNA from normal lymphocytes (NL) was used as a negative control for methylated sequences. Results shown are representative of those obtained from three independent experiments, and b-actin was used as a control. Values from pyrosequencing and GRT-PCR were determined from triplicates and are expressed as the mean \pm SEM. Significance of Mann-Whitney $U$ test, ${ }^{* *} p<0.01 ;{ }^{*} p<0.05$ 


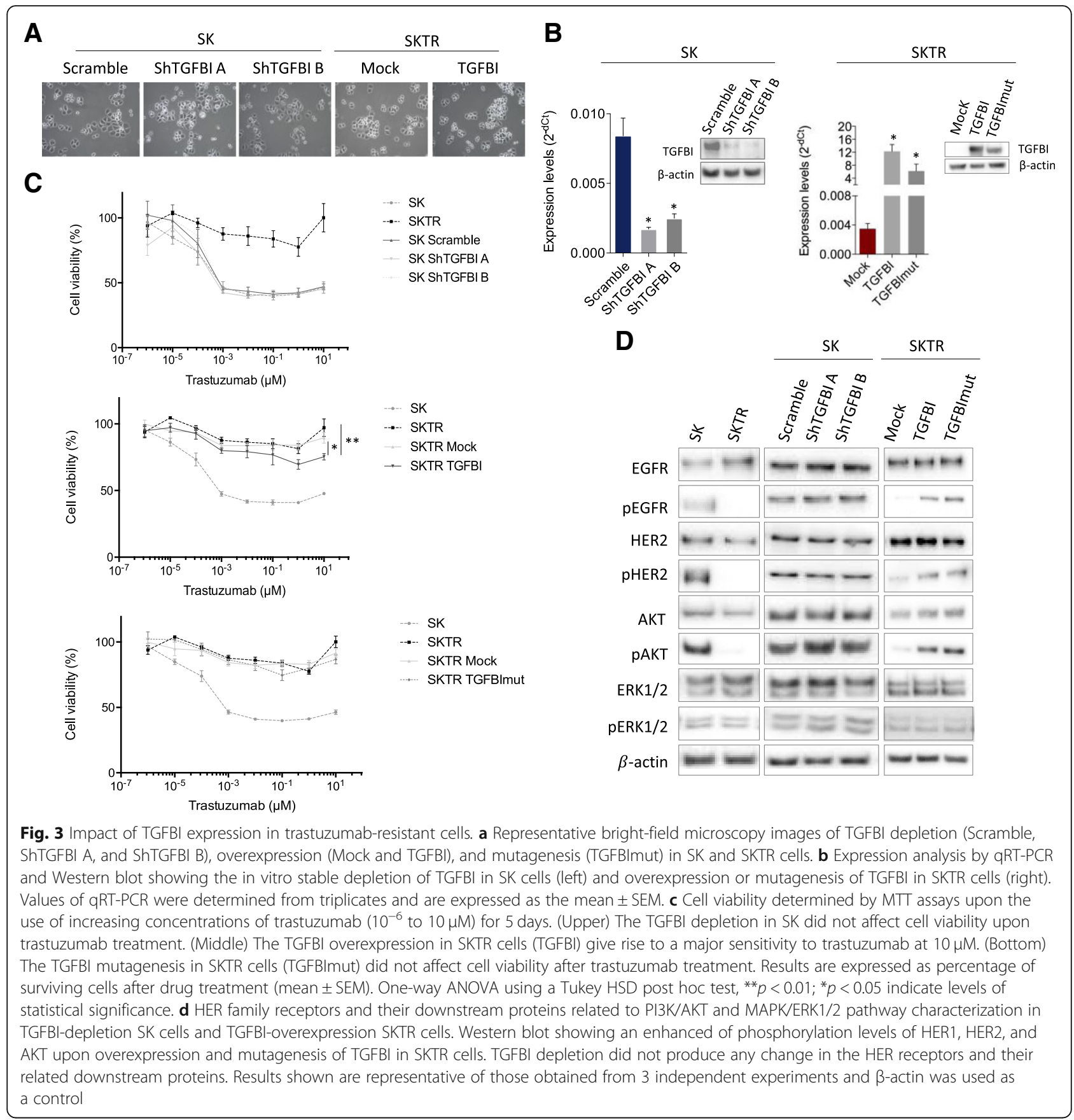

TGFBI hypermethylation in trastuzumab-resistant HER2+ breast cancer patients

Given the previous in vitro results obtained, we wanted to determine whether the presence of the TGFBI promoter CpG island hypermethylation-associated to trastuzumab resistance also occurring in $\mathrm{BC}$ patients. Therefore, we evaluated TGFBI gene methylation in human tumors from 24 HER2+ early BC patients as a preliminary study. As with our sensitive and resistant models, we analyzed TGFBI methylation by bisulfite pyrosequencing in pre- treatment samples from patients who were complete responders and non-responders to trastuzumab-based therapy (Fig. 4a). With the non-responder patients, we also evaluated TGFBI methylation after treatment (post-treatment samples), i.e., as we did in our SKTR model. For the non-response patients, we obtained paired samples (pretreatment and post-treatment samples) from 10 patients, pre-treatment samples only from 3 patients, and posttreatment samples only from 7 patients. For the patients with pathological complete response, only pre-treatment 
samples were evaluated. The limited number of samples obtained was, in part, due to the exhaustion of the sample during diagnostic procedures and the poor preservation of the DNA in the paraffin tissue (FFPE) blocks.

Our results showed similar pre-treatment TGFBI methylation levels $(p=0.651)$ in the patients with complete response to trastuzumab $(6.45 \% \pm 1.90)$ and in the nonresponders $(6.08 \% \pm 1.51$; Fig. $4 \mathrm{~b})$. These results indicate that the TGFBI methylation levels of the pre-treatment samples are not associated with the absence of response to trastuzumab. In contrast, the non-responsive patients showed significantly higher $(p=0.001)$ methylation levels of TGFBI in tumors following treatment with trastuzumab $(30.26 \% \pm$ $3.52)$ than before starting the therapy $(6.08 \% \pm 1.51)$, suggesting that acquired resistance to trastuzumab is associated with increased methylation levels of TGFBI. In particular, when considering the non-responsive patients with pre- and post-treatment paired samples, we observed a significant $(p<0.001)$ TGFBI promoter hypermethylation after trastuzumab ( 8 out of $10=80 \%$ ) compared to the pre-treatment samples, indicating that the increase in TGFBI methylation levels is associated with trastuzumab resistance (Fig. 4c). Importantly, the ROC curve analysis with an AUC of 0.95 ( $p<$ $0.0001 ; 95 \%$ CI 0.803 to 0.996 ) allowed us to clearly differentiate the methylation levels of TGFBI between the pre- and post-treatment samples of the non-responsive patients. This result suggests that TGFBI could be a potential biomarker for monitoring trastuzumab response during HER2+ $\mathrm{BC}$ patients' treatment (Fig. 4d). In addition, the HosmerLemeshow test (chi-square $=89,606 ; p=0.3456$ ) suggests that the methylation of TGFBI has a certain ability to predict the resistance to trastuzumab when compared with an ideal model (Additional file 4: Figure S3). On the other hand, no significant association between TGFBI methylation levels before and after treatment and the clinicalhistopathological characteristics was identified (Table 2).
A

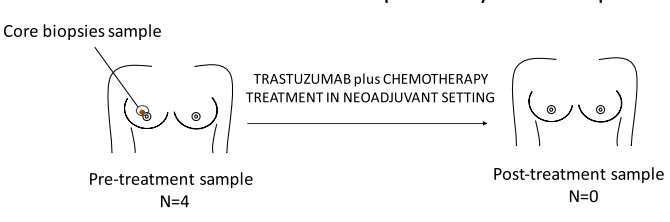

Trastuzumab resistant HER2-positive early breast cancer patients

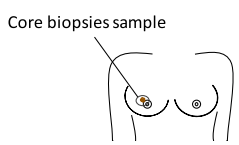

TRASTUZUMAB PlUS CHEMOTHERAPY TREATMENT IN NEOADJUVANT SETTING Pre-treatment sample $\mathrm{N}=13$

C

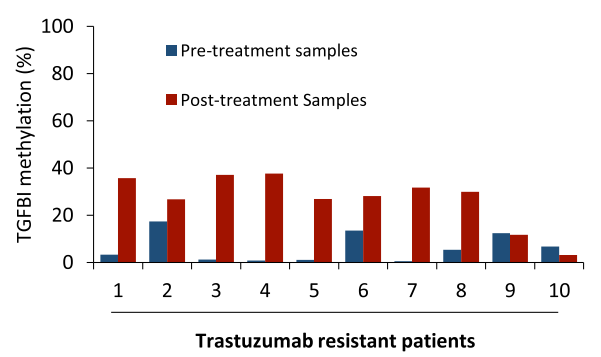

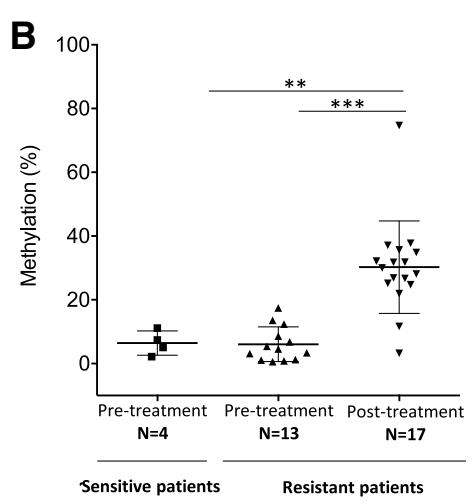

TGFBI

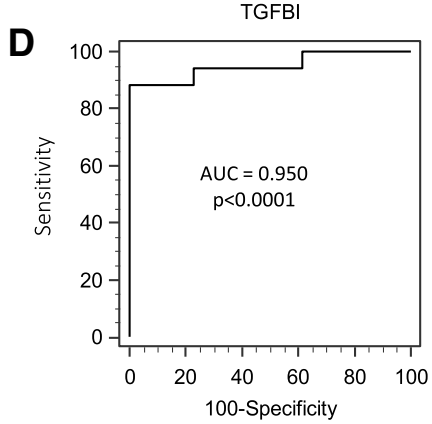

Fig. 4 TGFBI promoter hypermethylation in HER2+ breast cancer patients with sensitivity and resistance to trastuzumab. a Schematic representation of selected patient samples. TGFBI methylation levels were evaluated in tumor samples of 24 HER2+ breast cancer samples. From this cohort, after trastuzumab plus chemotherapy in neoadjuvant regimen, 20 patients developed partial or no response and 4 patients presented complete treatment response. Of the 20 patients with non-response, 10 patients had pre-treatment and post-treatment samples, 3 patients with pre-treatment only samples, and 7 with post-treatment only samples (Top). The 4 patients with complete response to treatment only had pretreatment samples (Bottom). b TGFBI methylation of 3 consecutive CpG sites in the $5^{\prime}$-end promoter CpG island in HER2+ breast cancer treated with trastuzumab analyzed by bisulfite pyrosequencing. The central solid line indicates the median and the limits of the vertical lines show the upper and lower percentiles. c TGFBI methylation of 3 consecutive CpG sites in the 5'-end promoter CpG island in resistant patients with paired pre- and post-treatment samples analyzed by bisulfite pyrosequencing. d Diagnostic accuracy of TGFBI hypermethylation for resistant HER2+ breast cancer samples. ROC analysis was applied to the TGFBI methylation levels analyzed by pyrosequencing for trastuzumab-resistant samples (pre- and post-treatment samples). Area under the curve (AUC) was 0.9502 ( $p<0.0001)$. TGFBl showed great potential for monitoring trastuzumab response in HER2+ breast cancer patients. Significance of Mann-Whitney $U$ test is indicated as ${ }^{* * *} p<0.001 ;{ }^{* *} p<0.01$ 


\section{Discussion}

HER2 is overexpressed in $20-30 \%$ of BC and is associated with aggressive phenotype and poor prognosis. Despite the initial good response to the treatment, a large portion of patients present de novo or acquired treatment resistance. Currently, HER2 detection is the only validated biomarker for predicting the benefit of antiHER2 therapies [33]. While different cellular and molecular mechanisms involved in trastuzumab resistance have been described, none of them are used to detect, predict, or monitor BC treatment in a clinical routine [7, $8,34]$. Thus, there is great interest in finding potential

Table 2 Clinical and pathological characteristics in HER2positive early breast cancer according to TGFBI promoter methylation levels

\begin{tabular}{|c|c|c|c|}
\hline \multirow[t]{2}{*}{ Characteristics } & \multicolumn{2}{|c|}{$\begin{array}{l}\% \\
N=10\end{array}$} & \multirow[t]{2}{*}{$\begin{array}{l}p \\
\text { value* }\end{array}$} \\
\hline & $n$ & Median (p25-p75) & \\
\hline Age & 10 & $-0.383^{*}$ & $0.275^{* 1}$ \\
\hline Menopause & & & $0.151^{* 2}$ \\
\hline Premenopausal & 5 & $32.13(24.58-35.86)$ & \\
\hline Postmenopausal & 5 & $14.58(-0.68-25.77)$ & \\
\hline ER & & & $0.089^{* 2}$ \\
\hline Negative & 2 & $2.85(-3.57-9.28)$ & \\
\hline Positive & 8 & $28.50(19.58-34.00)$ & \\
\hline HER2 status & & & - \\
\hline $\mathrm{FISH}$ & 0 & - & \\
\hline $\mathrm{IHC}+$ & 10 & $25.18(9.28-32.13)$ & \\
\hline Histological grade & & & - \\
\hline Grade 1-2 & 10 & $25.18(9.28-32.13)$ & \\
\hline Grade 3 & 0 & - & \\
\hline Clinical stage & & & $0.804^{* 3}$ \\
\hline$\| \mathrm{B}$ & 2 & $16.15(-3.57-25.86)$ & \\
\hline$\| I A$ & 2 & $19.58(14.58-24.58)$ & \\
\hline$\| \mathrm{IIB}$ & 6 & $28.50(9.28-32.13)$ & \\
\hline Pathological response & & & - \\
\hline No response & 1 & 9.28 & \\
\hline Partial response & 9 & $25.77(14.58-32.13)$ & \\
\hline Percentage of tumor shrinkage ${ }^{* *}$ & 10 & 0.029 & $0.957^{* 1}$ \\
\hline Miller \& Payne & & & $1.000^{* 2}$ \\
\hline $\mathrm{G} 2$ & 1 & 0.68 & \\
\hline G3 & 2 & $22.57(9.28-35.86)$ & \\
\hline G4 & 7 & 25.77 (19.58-31.68) & \\
\hline Type of surgery & & & $0.267^{* 2}$ \\
\hline Lumpectomy & 2 & $10.51(-3.57-24.58)$ & \\
\hline Mastectomy & 8 & $28.50(11.93-34.00)$ & \\
\hline
\end{tabular}

${ }^{* 1}$ Spearman correlation

${ }^{* 2}$ Mann-Whitney test

$*{ }^{3}$ Kruskal-Wallis test biomarkers able to detect, predict, or monitor trastuzumab response which, in turn, would allow us to stratify patients and save costs and toxicities for those who do not respond to treatment.

Epigenetic biomarker studies are focused on analyzing the methylation changes in the promoter regions of candidate genes in specific tumor types for the implication in gene silencing $[9,34]$. In general, this gene repression results in an adaptive advantage for the cells, allowing a more aggressive and invasive phenotype to be adopted. After analyzing the promoter methylation profile of longterm trastuzumab-sensitive and trastuzumab-resistant models developed in our laboratory $[16,17]$, three different methylated genes (TGFBI, CXCL2, and SLC38A1) implicated in cancer were identified and subsequently validated using different methylation and expression approaches. Additionally, the selected genes were validated in two other long-term trastuzumab-sensitive (AU) and trastuzumab-resistant (AUTR) human HER2+ BC cell lines developed by our group [17]. Although both cell lines analyzed derive from the same patient and have similar genetic and transcriptomic profiles, they have some differences in transcriptional levels [35]. Moreover, SKBr3 and AU565 have been described as having distinct responses to drug treatment depending on culture conditions [36]. From all the genes selected, TGFBI (also known as Big-H3 or keratoepithelin) was the only one with a consistent methylation, expression, and protein pattern in both resistant models. This epigenetic silencing of the TGFBI gene observed was in line with previous studies of other cancers [37, 38].

As explained before, TGFBI encodes a $68-\mathrm{kDa}$ secretory protein induced by transforming growth factor- $\beta$ (TGF- $\beta$ ) in human adenocarcinoma cells as well as other human cell types [39]. According to the bibliography, TGFBI protein is composed by 683 amino acids containing a secretory signal (SP) in the $N$-terminal cysteine-rich domain (CRD), and four fasciclin-1 domains (FAS1-1, FAS12, FAS1-3, and FAS1-4), which contain several known integrin-binding motifs including NKDIL, YH18, and EPDIM as well as an Arg-Gly-Asp (RGD) domain [22]. TGFBI has been reported to function as an ECM protein to mediate cell adhesion and migration through interacting with collagen, fibronectin, and laminin and several integrins including $\alpha 1 \beta 1, \alpha 3 \beta 1, \alpha v \beta 3$, and $\alpha v \beta 5$ [30-32]. While TGFBI has been reported to be involved in tumorigenesis, its role is not clear. In this paper, we have linked the promoter DNA methylation-associated silencing of TGFBI with a possible tumor suppressor function in trastuzumab-resistant models. Several previous reports have indicated that TGFBI plays the role of a tumor suppressor gene in various cancers such as lung, breast, and ovarian [25, 40-42]. However, in other cancers, such as colon or pancreas, TGFBI has been described as having a 
tumor-promoting function [43-46]. These opposing effects of TGFBI suggest that its expression and function are dependent on cell type [22].

TGFBI promotes cell adhesion by interacting with different integrins, which, in turn, has been shown to play an important role in tumor progression in humans. TGFBI's inhibition of invasive capacity correlates with a previous analysis by our group, which revealed a greater invasive capacity for SKTR compared to the SK [16]. To determine if TGFBI has a potential role in trastuzumab resistance, different functional studies were carried out. Our results show that TGFBI overexpression in SKTR significantly sensitizes the cells to the treatment and activates some HER2 downstream protein pathways (pAKT, pHER1, and pHER2), adopting a similar pattern to SK. In contrast, TGFBI knockdown in TGFBI endogenous expressed in the SK cells did not produce any effect. A direct interaction between the HER2 receptor and different integrins has been described in multiple reports [47, 48]. Moreover, different integrins have been related to trastuzumab resistance such as $\beta 1$ or $\alpha 6 \beta 4$ $[49,50]$. Therefore, TGFBI mutated vector was performed to investigate the role of the RGD motif and the three integrin-binding motifs NKDIL, EPDIM, and YH18 in trastuzumab response [22]. Like TGFBI overexpression, the TGFBI mutated form induced the activation of different HER2 downstream proteins, but there were no differences in trastuzumab treatment response. Therefore, it is likely that the four mutated domains of TGFBI are required for its function in trastuzumab response, which is consistent with other studies that described the involvement of FAS1 or RGD motifs in tumor angiogenesis and tumor growth inhibition, as well as in promoting apoptosis [31, 51-53]. Moreover, it has also been described that TGFBI is involved in mesothelioma progression through the AKT/ mTOR pathway which could be related to the HER2 pathway changes observed in TGFBI overexpression and mutagenesis [54]. In summary, this study suggests that TGFBI expression may promote the effectiveness of trastuzumab treatment.

The methylation of TGFBI showed certain capacity to predict the resistance to trastuzumab according to the Hosmer-Lemeshow test; however, these results should be interpreted with caution because the number of grouped patients was small. Although more in vitro functional analysis are necessary to elucidate the TGFBI role in trastuzumab resistance, we suggest some hypothetical situations taking into account all TGFBI action mechanisms. It has been described that TGFBI could inhibit cell adhesion to various ECM proteins inhibiting cell proliferation and invasion in neuroblastoma [55]. Moreover, cell adhesion to ECM has been consistently reported as one of the mechanisms used by tumor cells to resist chemotherapy [56]. These observations are in correlation with previous studies by our group, where it is exposed that our SKTR model presented a high significant adhesion capacity to bind to extracellular matrix proteins like fibronectin, collagen I, collagen IV, or laminin I compared to SK [16]. Therefore, we hypothesized that in the absence of TGFBI, the integrins could bind to the ECM providing attachment sites for cells inducing more invasion, or acting as a physical drug barrier, restricting drug transport and limiting their efficacy. The intratumoral diffusion and physical masking of HER receptors by ECM proteins have also been described as a mechanism that can affect the therapeutic efficacy of some drugs including trastuzumab [57].

Finally, we correlated the previous in vitro results in human samples treated with neoadjuvant anthracyclinetaxane-based chemotherapy plus trastuzumab. In our preliminary study, a higher TGFBI methylation level after treatment was observed in patients who developed treatment resistance. These results are in accordance with other studies which demonstrated an association between TGFBI hypermethylation and poor prognosis in prostate and lung cancer [41]. Moreover, decreased TGFBI expression was identified in advanced stages of BC and NSCLC tumors $[40,58]$. Due to the small size of the patient cohort and the retrospective design, the role of TGFBI methylation as a biomarker requires further validation in a larger and independent cohort. Nevertheless, the present study brings to light for the first time that TGFBI methylation has a significant discriminative value between pre- and post-treatment samples, as demonstrated by ROC curve analyses. Interestingly, the TGFBI promoter methylation analysis in responder patients showed similar methylation levels with pre-treatment samples of non-responders. This observation suggests a possible role of TGFBI as a monitoring biomarker for trastuzumab response in patients with HER2+ BC.

Although no significant association was found between TGFBI methylation before and after treatment and patients' clinical-histopathologic characteristics, a high number ER-positive patients were observed. In this sense, previous studies have shown that there is crosstalk between ER and HER2 pathways and it affects the response to treatment [59]. In addition, although the type of surgery is not associated with a higher TGFBI methylation level in post-treatment samples, $73 \%$ of our patients were treated with mastectomy versus $27 \%$ with lumpectomy, probably due to the high prevalence of stage III. The low histological grade and high clinical stage to treatment were probably due to most of the patients having non-operable cancers including inflammatory tumors. Currently, neoadjuvant treatment is generally used for operable HER2+ BC thanks to an improvement in the efficacy of the drugs used for treatment [60]. At the time our cohort was 
treated, none of these drugs were yet available. That said, trastuzumab remains the gold standard treatment for HER2+ BC.

\section{Conclusions}

The results obtained in this study have provided an overview of the DNA methylation pattern in HER2+resistant BC. TGFBI promoter hypermethylation could be a potential methylation monitoring biomarker for trastuzumab response. In addition, once the resistance is developed, demethylation of TGFBI with compounds that inhibit DNA methyltransferases could contribute to the sensitization of breast cancer cells to the trastuzumab treatment. However, further studies are required to identify the specific role TGFBI plays in trastuzumab resistance in the neoadjuvant settings to definitively show $T G F B I$ as a clinical relevant biomarker. Future studies based on TGFBI promoter methylation analyses in circulating DNA during neoadjuvant treatment could be a potential strategy with which to confirm our findings.

\section{Additional files}

\section{Additional file 1: Additional methods. (DOCX $30 \mathrm{~kb}$ )}

Additional file 2: Figure S1. DNA methylation-associated silencing of CXCL2 and SLC38A1 comparing the trastuzumab-resistant (AUTR) and -sensitive (AU) AU565 cell model. (A) Expression levels of CXCL2 and SLC38A1 in the unmethylated (AU) and methylated (AUTR) models determined by qRT-PCR. (B) Restored expression of CXCL2 and SLC38A1 after DNA demethylating agent 5-aza-2'-deoxycytidine (5-aza- $d C$ ) in the AUTR methylated cell line by qRT-PCR. (C) DNA methylation levels of CXCL2 and SLC38A1 in AU and AUTR cell lines by methylation-specific polymerase chain reaction (MSP) analysis. (D) Protein expression of CXCL2 and SLC38A1 in AU and AUTR cells before and after 5-aza-dC treatment by Western blot. In MSP, the presence of visible polymerase chain reaction products in lanes marked $U$ indicate unmethylated sequences; the presence of products in lanes marked $M$ indicate methylated sequences. In vitro methylated DNA (IVD) was used as a positive control for methylated sequences. DNA from normal lymphocytes $(\mathrm{NL})$ was used as a negative control for methylated sequences. Results shown are representative of those obtained from three independent experiments and b- actin was used as a control. Values from pyrosequencing and qRT-PCR were determined from triplicates and are expressed as the mean \pm SEM. Significance of Mann-Whitney $U$ test, ${ }^{* *} p<0.01$; ${ }^{*} p<0.05$. (PDF $235 \mathrm{~kb}$ )

Additional file 3: Figure S2. Cell viability determined by MTT assays upon trastuzumab treatment for 5 days. (A) TGFBI depletion in SK cells, (B) TGFBI overexpression in SKTR cells (TGFBI) and (C) TGFBI-mutagenesis in SKTR cells (TGFBImut). The continuous line represents the fitted doseresponse curve while discontinuous lines indicate the simultaneous 95\% confidence interval $(\mathrm{Cls})$ for the continuous line with the same color. ANOVA using a Tukey HSD post hoc test, ${ }^{* * P}<0.01$; ${ }^{*} \mathrm{P}<0.05$ indicate levels of statistical significance. (PDF $397 \mathrm{~kb}$ )

Additional file 4: Figure S3. Calibration curve for the reliability of TGFBI to predict trastuzumab resistance. Calibration was assessed using the Hosmer-Lemeshow test and graphically using a calibration plot. The observed frequency was calculated for each group. Predicted probability refers to the predicted probabilities generated by the model. Patients were grouped according to deciles of the predicted probabilities. Grouped patients refers to patients grouped at each decile of predicted probabilities generated by the model. A LOWESS regression was fit to all data points (blue line). (PDF $119 \mathrm{~kb}$ )

\section{Abbreviations}

5-aza-dC: 5-Aza-2'-deoxycytidine; Akt: Protein kinase B; BC: Breast cancer; CXCL2: C-X-C motif chemokine ligand 2; ECM: Extracellular matrix; FDR: False discovery rate; GO: Gene ontology; HER: Human epidermal growth factor receptor family; HER2: Human epidermal growth factor receptor 2; HER2+: HER2-positive; MAPK: Ras-mitogen-activated protein kinases; MSP: Methylation-specific PCR; PI3K: Phosphatidylinositol 3'-kinase; SLC38A1: Solute carrier Family 38 Member 1; TGF- $\beta$ : Transforming growth factor beta; TGFBI: Transforming growth factor beta induced

\section{Acknowledgements}

The authors thank Aitor Rodriguez-Casanova (Translational Medical Oncology (Oncomet), Health Research Institute of Santiago (IDIS), University Clinical Hospital of Santiago (CHUS), Santiago de Compostela, Spain) for his kind support in the methylation analysis. They also thank Maria Buxó (Girona Biomedical Research Institute (IDIBGI), Girona, Spain) and Marc Saez (CIBER of Epidemiology and Public Health (CIBERESP), Madrid, Spain and Research Group on Statistics, Econometrics and Health (GRECS), University of Girona, Girona, Spain) for assistance with the statistical analysis. We thank the Genomics and Epigenomics Service from the Cancer Epigenetics and Biology Program (PRBC), Bellvitge Biomedical Research Institute (IDIBELL)], the Huntsman Cancer Foundation, and the High Throughput Genomics and Bioinformatic Analysis Shared Resource (HTGBA) at Huntsman Cancer Institute (HCl) for their support. HTGBA is supported by $\mathrm{HCl}$ Cancer Center Support Grant (5P30CA042014-24; the content is solely the responsibility of the authors and does not necessarily represent the official views of the U.S. National Cancer Institute or the National Institutes of Health).

\section{Authors' contributions}

TP and ME conceived and designed the study. SP, AD-L, FS, HJF, DHL, ALW, GO, ME, and TP developed the methodology. SP, AD-L, DHL, ALW, GV, AH, $T P$, and ME contributed to data acquisition (provided facilities etc.). SP, AD- L, $A B C, G V$, and $A H$ participated in data analysis and interpretation. SP, AD-L, GV, FS, HJF, ABC, DL, ALW, TP, and ME wrote, reviewed, and/or revised the manuscript. SP, GV, AH, DL, and ALW participated in administrative, technical, or material support (i.e., reporting or organizing data, construction database). TP and ME supervised the study. All authors read and approved the final manuscript.

\section{Funding}

This work was supported in part by the Spanish Instituto de Salud Carlos III (ISCIII; FIS PI11/00692 and PI14/00329; to' T. Puig), Fundacion Ramon Areces (to T. Puig), the support of Catalonian Government (2017SGR00385), and La Marató de TV3 (20131530, TPuig), financial support was from the University of Girona (MPCUdG2016/036), and the University of Girona and La Caixa Foundation awarded S. Palomeras with a predoctoral grant. A.D.-L. is funded by a "Juan Rodés" contract (JR17/00016) from ISCIII. Ana B Crujeiras is funded by a research contract "Miguel Servet" (CP17/00088) from the ISCIII.

\section{Availability of data and materials}

The microarray and sequencing dataset generated during the current study is available in NCBI GEO (www.ncbi.nlm.nih.gov/geo) under accession GSE123754 and GSE114575, respectively.

\section{Ethics approval and consent to participate}

This study was performed in compliance with the clinical research guidelines established by the Dr. Josep Trueta University Hospital Ethics Committee. Signed informed consent was obtained from each patient.

\section{Consent for publication}

Not applicable.

\section{Competing interests}

The authors declare that they have no competing interests.

\section{Author details}

${ }^{1}$ New Therapeutics Targets Lab (TargetsLab), Department of Medical Sciences, University of Girona, E-17071 Girona, Catalonia, Spain. ${ }^{2}$ Cancer Epigenetics and Biology Program (PEBC), Bellvitge Biomedical Research Institute (IDIBELL), Hospitalet de Llobregat, Barcelona, Catalonia, Spain. ${ }^{3}$ Cancer Epigenomics, Translational Medical Oncology (Oncomet), Health 
Research Institute of Santiago (IDIS), University Clinical Hospital of Santiago(CHUS/SERGAS), CIBERONC, Santiago de Compostela, Spain. ${ }^{4}$ Medical Oncology Department, Catalan Institute of Oncology (ICO), Girona, Catalonia, Spain. ${ }^{5}$ Girona Biomedical Research Institute (IDIBGI), E-17071 Girona, Catalonia, Spain. ${ }^{6}$ Pathology Department, Dr. Josep Trueta Hospital and Catalan Institute of Health (ICS), E-17071 Girona, Catalonia, Spain. ' Laboratory of Epigenomics in Endocrinology and Nutrition, Health Research Institute of Santiago (IDIS), University Clinical Hospital of Santiago (CHUS/SERGAS), Santiago de Compostela, Spain. ${ }^{8} \mathrm{CIBER}$ Fisiopatologia de la Obesidad y Nutricion (CIBERobn), Santiago de Compostela, Spain. ${ }^{9}$ Department of Oncological Sciences, Huntsman Cancer Institute, University of Utah, Salt Lake City, USA. ${ }^{10} \mathrm{Centro}$ de Investigacion Biomedica en Red Cancer (CIBERONC), Madrid, Spain. ${ }^{11}$ Physiological Sciences Department, School of Medicine and Health Sciences, University of Barcelona (UB), Barcelona, Catalonia, Spain. ${ }^{12}$ Institucio Catalana de Recerca i Estudis Avançats (ICREA), Barcelona, Catalonia, Spain. ${ }^{13}$ Josep Carreras Leukaemia Research Institute (IJC), Badalona, Barcelona, Catalonia, Spain.

\section{Received: 15 January 2019 Accepted: 6 June 2019}

\section{Published online: 05 July 2019}

\section{References}

1. Global Burden of Disease Cancer Collaboration, Fitzmaurice C, Allen C, Barber RM, Barregard L, Bhutta ZA, et al. Global, regional, and national cancer incidence, mortality, years of life lost, years lived with disability, and disabilityadjusted life-years for 32 cancer groups, 1990 to 2015: a systematic analysis for the global burden of disease study. JAMA Oncol. 2017;3(4):524-48.

2. Bauer $K$, Parise $C$, Caggiano V. Use of ER/PR/HER2 subtypes in conjunction with the 2007 St Gallen consensus statement for early breast cancer. BMC Cancer. 2010;10(1):228.

3. Hudis CA. Trastuzumab--mechanism of action and use in clinical practice. $\mathrm{N}$ Engl J Med. 2007:357(1):39-51.

4. Luque-Cabal M, García-Teijido P, Fernández-Pérez Y, Sánchez-Lorenzo L, Palacio-Vázquez I. Mechanisms behind the resistance to trastuzumab in HER2-amplified breast cancer and strategies to overcome it. Clin Med Insights Oncol. 2016;10(Suppl 1):21-30.

5. Nakashoji A, Hayashida T, Yokoe T, Maeda H, Toyota T, Kikuchi M, et al. The updated network meta-analysis of neoadjuvant therapy for HER2-positive breast cancer. Cancer Treat Rev. 2018;62:9-17.

6. Carter P, Presta L, Gorman CM, Ridgway JB, Henner D, Wong WL, et al. Humanization of an anti-p185HER2 antibody for human cancer therapy. Proc Natl Acad Sci U S A. 1992;89(10):4285-9.

7. Nahta R, Yu D, Hung M-C, Hortobagyi GN, Esteva FJ. Mechanisms of disease: understanding resistance to HER2-targeted therapy in human breast cancer. Nat Clin Pract Oncol. 2006;3(5):269-80.

8. Scaltriti M, Rojo F, Ocaña A, Anido J, Guzman M, Cortes J, et al. Expression of p95HER2, a truncated form of the HER2 receptor, and response to antiHER2 therapies in breast cancer. J Natl Cancer Inst. 2007:99(8):628-38.

9. Esteller M. Epigenetics in Cancer. N Engl J Med. 2008;358(11):1148-59.

10. Paluszczak J, Baer-Dubowska W. Epigenetic diagnostics of cancer - the application of DNA methylation markers. J Appl Genet. 2006;47(4):365-75.

11. Duffy MJ, Napieralski R, Martens JWM, Span PN, Spyratos F, Sweep FCGJ, et al. Methylated genes as new cancer biomarkers. Eur J Cancer. 2009;45(3):335-46.

12. Rice JC, Ozcelik H, Maxeiner P, Andrulis I, Futscher BW. Methylation of the BRCA1 promoter is associated with decreased BRCA1 mRNA levels in clinical breast cancer specimens. Carcinogenesis. 2000;21(9):1761-5.

13. Dammann R, Schagdarsurengin U, Strunnikova M, Rastetter M, Seidel C, Liu $L$, et al. Epigenetic inactivation of the Ras-association domain family 1 (RASSF1A) gene and its function in human carcinogenesis. Histol Histopathol. 2003;18(2):665-77.

14. Jones PA. Functions of DNA methylation: islands, start sites, gene bodies and beyond. Nat Rev Genet. 2012;13(7):484-92.

15. Trempe GL. Human breast cancer in culture. Recent Results Cancer Res. 1976;57(57):33-41

16. Blancafort A, Giró-Perafita A, Oliveras G, Palomeras S, Turrado C, Campuzano O, et al. Dual fatty acid synthase and HER2 signaling blockade shows marked antitumor activity against breast cancer models resistant to antiHER2 drugs. PLoS One. 2015;10(6):e0131241.

17. Puig T, Aguilar H, Cufí S, Oliveras G, Turrado C, Ortega-gutiérrez S, et al. A novel inhibitor of fatty acid synthase shows activity against HER2 + breast cancer xenografts and is active in anti-HER2 drug-resistant cell lines. Breast Cancer Res. 2011;13(6):R131.

18. Edge SB, Compton CC. The American Joint Committee on Cancer: the 7th edition of the AJCC Cancer staging manual and the future of TNM. Ann Surg Oncol. 2010;17(6):1471-4.

19. Bender CM, Pao MM, Jones PA. Inhibition of DNA methylation by 5-aza-2'deoxycytidine suppresses the growth of human tumor cell lines. Cancer Res. 1998;58(1):95-101.

20. Sandoval J, Heyn H, Moran S, Serra-Musach J, Pujana MA, Bibikova M, et al. Validation of a DNA methylation microarray for 450,000 CpG sites in the human genome. Epigenetics. 2011;6(6):692-702.

21. Naumov VA, Generozov EV, Zaharjevskaya NB, Matushkina DS, Larin AK, Chernyshov SV, et al. Genome-scale analysis of DNA methylation in colorectal cancer using Infinium HumanMethylation450 BeadChips. Epigenetics. 2013:8(9):921-34.

22. Ween MP, Oehler MK, Ricciardelli C. Transforming growth factor-betainduced protein $(\mathrm{TGFBI}) /($ Big-H3): a matrix protein with dual functions in ovarian cancer. Int J Mol Sci. 2012;13(8):10461-77.

23. Diaz-Lagares A, Crujeiras AB, Lopez-Serra P, Soler M, Setien F, Goyal A, et al. Epigenetic inactivation of the p53-induced long noncoding RNA TP53 target 1 in human cancer. Proc Natl Acad Sci. 2016;113(47):E7535-44.

24. Diaz-Lagares A, Mendez-Gonzalez J, Hervas D, Saigi M, Pajares MJ, Garcia D, et al. A novel epigenetic signature for early diagnosis in lung cancer. Clin Cancer Res. 2016;22(13):3361-71.

25. Wen G, Partridge MA, Li B, Hong M, Liao W, Cheng SK, et al. TGFBI expression reduces in vitro and in vivo metastatic potential of lung and breast tumor cells. Cancer Lett. 2011:308(1):23-32.

26. Kronski E, Fiori ME, Barbieri O, Astigiano S, Mirisola V, Killian PH, et al. miR181b is induced by the chemopreventive polyphenol curcumin and inhibits breast cancer metastasis via down-regulation of the inflammatory cytokines CXCL1 and -2. Mol Oncol. 2014;8(3):581-95.

27. Wang $K$, Cao F, Fang W, Hu Y, Chen Y, Ding H, et al. Activation of SNAT1/ SLC38A1 in human breast cancer: correlation with p-Akt overexpression. BMC Cancer. 2013;13:343.

28. Dejeux E, El Abdalaoui H, Gut IG, Tost J. Identification and quantification of differentially methylated loci by the Pyrosequencing ${ }^{\text {TM }}$ technology. Methods Mol Biol. 2009;507:189-205.

29. Herman JG, Graff JR, Myohanen S, Nelkin BD, Baylin SB. Methylation-specific PCR: a novel PCR assay for methylation status of CpG islands (DNA methylation/tumor suppressor genes/pl6/p15). Proc Natl Acad Sci U S A 1996;93:9821-6

30. Hashimoto K, Noshiro M, Ohno S, Kawamoto T, Satakeda H, Akagawa Y, et al. Characterization of a cartilage-derived 66-kDa protein (RGD-CAP/ßig-h3) that binds to collagen. Biochim Biophys Acta - Mol Cell Res. 1997;1355(3):303-14.

31. Kim J-E, Jeong H-W, Nam J-O, Lee B-H, Choi J-Y, Park R-W, et al. Identification of motifs in the fasciclin domains of the transforming growth factor- $\beta$-induced matrix protein $\beta i g-h 3$ that interact with the av $\beta 5$ integrin. J Biol Chem. 2002;277(48):46159-65.

32. Billings PC, Charles Whitbeck J, Adams CS, Abrams WR, Cohen AJ, Engelsberg $\mathrm{BN}$, et al. The transforming growth factor- $\beta$-inducible matrix protein Big-h3 interacts with fibronectin. J Biol Chem. 2002;277(31):28003-9.

33. Slamon DJ, Leyland-Jones B, Shak S, Fuchs H, Paton V, Bajamonde A, et al. Use of chemotherapy plus a monoclonal antibody against HER2 for metastatic breast cancer that overexpresses HER2. N Engl J Med. 2001;344(11):783-92.

34. Nahta $R$, Yuan $L X H H$, Zhang $B$, Kobayashi R, Esteva FJ. Insulin-like growth factor-I receptor/human epidermal growth factor receptor 2 heterodimerization contributes to trastuzumab resistance of breast cancer cells. Cancer Res. 2005;65(23):11118-28.

35. Neve RM, Chin K, Fridlyand J, Yeh J, Baehner FL, Fevr T, et al. A collection of breast cancer cell lines for the study of functionally distinct cancer subtypes. Cancer Cell. 2006;10(6):515-27.

36. Weigelt B, Lo AT, Park CC, Gray JW, Bissell MJ. HER2 signaling pathway activation and response of breast cancer cells to HER2-targeting agents is dependent strongly on the 3D microenvironment. Breast Cancer Res Treat. 2010;122(1):35-43

37. Shao G, Berenguer J, Borczuk AC, Powell CA, Hei TK, Zhao Y. Epigenetic inactivation of Betaig-h3 gene in human cancer cells. Cancer Res. 2006; 66(9):4566-73.

38. Wang N, Zhang H, Yao Q, Wang Y, Dai S, Yang X. TGFBI promoter hypermethylation correlating with paclitaxel chemoresistance in ovarian cancer. J Exp Clin Cancer Res. 2012;31(1):6. 
39. Skonier J, Neubauer M, Madisen L, Bennett K, Plowman GD, Purchio AF. cDNA cloning and sequence analysis of Big-h3, a novel gene induced in a human adenocarcinoma cell line after treatment with transforming growth factor- $\beta$. DNA Cell Biol. 1992;11(7):511-22.

40. Calaf GM, Echiburú-Chau C, Zhao YL, Hei TK. BigH3 protein expression as a marker for breast cancer. Int J Mol Med. 2008:21(5):561-8.

41. Shah JN, Shao G, Hei TK, Zhao Y. Methylation screening of the TGFBI promoter in human lung and prostate cancer by methylation-specific PCR. BMC Cancer. 2008;8(1):284.

42. Ahmed AA, Mills AD, Ibrahim AEKK, Temple J, Blenkiron C, Vias M, et al. The extracellular matrix protein TGFBI induces microtubule stabilization and sensitizes ovarian cancers to paclitaxel. Cancer Cell. 2007;12(6):514-27.

43. Kitahara O, Furukawa Y, Tanaka T, Kihara C, Ono K, Yanagawa R, et al. Alterations of gene expression during colorectal carcinogenesis revealed by cDNA microarrays after laser-capture microdissection of tumor tissues and normal epithelia. Cancer Res. 2001;61(9):3544-9.

44. Schneider D, Kleeff J, Berberat PO, Zhu Z, Korc M, Friess H, et al. Induction and expression of betaig-h3 in pancreatic cancer cells. Biochim Biophys Acta. 2002;1588(1):1-6.

45. Guo S-K, Shen M-F, Yao H-W, Liu Y-S. Enhanced expression of TGFBI promotes the proliferation and migration of glioma cells. Cell Physiol Biochem. 2018;49(3):1097-109.

46. Chen W-Y, Tsai Y-C, Yeh H-L, Suau F, Jiang K-C, Shao A-N, et al. Loss of SPDEF and gain of TGFBI activity after androgen deprivation therapy promote EMT and bone metastasis of prostate cancer. Sci Signal. 2017; 10(492):eaam6826.

47. Desgrosellier JS, Cheresh DA. Integrins in cancer: biological implications and therapeutic opportunities. Nat Rev Cancer. 2010;10(1):9-22.

48. Soung YH, Clifford JL, Chung J. Crosstalk between integrin and receptor tyrosine kinase signaling in breast carcinoma progression. BMB Rep. 2010; 43(5):311-8.

49. Lesniak D, Xu Y, Deschenes J, Lai R, Thoms J, Murray D, et al. 1-integrin circumvents the antiproliferative effects of trastuzumab in human epidermal growth factor receptor-2-positive breast cancer. Cancer Res. 2009;69(22):8620-8.

50. Guo W, Pylayeva Y, Pepe A, Yoshioka T, Muller WJ, Inghirami G, et al. $\beta 4$ integrin amplifies ErbB2 signaling to promote mammary tumorigenesis. Cell. 2006;126(3):489-502

51. Nam J-O, Jeong H-W, Lee B-H, Park R-W, Kim I-S. Regulation of tumor angiogenesis by fastatin, the fourth FAS1 domain of Big-h3, via avß3 integrin. Cancer Res. 2005;65(10):4153-61.

52. Zhao YL, Piao CQ, Hei TK. Overexpression of Betaig-h3 gene downregulates integrin alpha5beta1 and suppresses tumorigenicity in radiation-induced tumorigenic human bronchial epithelial cells. $\mathrm{Br} \mathrm{J}$ Cancer. 2002;86(12):1923-8.

53. Kim J-E, Kim S-J, Jeong H-W, Lee B-H, Choi J-Y, Park R-W, et al. RGD peptides released from $\beta$ ig-h3, a TGF- $\beta$-induced cell-adhesive molecule, mediate apoptosis. Oncogene. 2003;22(13):2045-53.

54. Wen G, Hong M, Li B, Liao W, Cheng SK, Hu B, et al. Transforming growth factor- $\beta$-induced protein (TGFBI) suppresses mesothelioma progression through the Akt/mTOR pathway. Int J Oncol. 2011;39(4):1001-9.

55. Becker J, Volland S, Noskova I, Schramm A, Schweigerer LL, Wilting J. Keratoepithelin reverts the suppression of tissue factor pathway inhibitor 2 by MYCN in human neuroblastoma: a mechanism to inhibit invasion. Int J Oncol. 2008;32(1):235-40.

56. Bissey P-A, Law JH, Bruce JP, Shi W, Renoult A, Chua MLK, et al. Dysregulation of the MiR-449b target TGFBI alters the TGF $\beta$ pathway to induce cisplatin resistance in nasopharyngeal carcinoma. Oncogenesis. 2018;7(5):40

57. Beyer I, Li Z, Persson J, Liu Y, van Rensburg R, Yumul R, et al. Controlled extracellular matrix degradation in breast cancer tumors improves therapy by trastuzumab. Mol Ther. 2011;19(3):479-89.

58. Pajares MJ, Agorreta J, Salvo E, Behrens C, Wistuba II, Montuenga LM, et al. TGFBI expression is an independent predictor of survival in adjuvant-treated lung squamous cell carcinoma patients. Br J Cancer. 2014;110(6):1545-51

59. Schettini F, Buono G, Cardalesi C, Desideri I, De Placido S, Del Mastro L. Hormone receptor/human epidermal growth factor receptor 2-positive breast cancer: where we are now and where we are going. Cancer Treat Rev. 2016;46:20-6.
60. Gianni L, Pienkowski T, Im Y-H, Tseng L-M, Liu M-C, Lluch A, et al. 5-year analysis of neoadjuvant pertuzumab and trastuzumab in patients with locally advanced, inflammatory, or early-stage HER2-positive breast cancer (NeoSphere): a multicentre, open-label, phase 2 randomised trial. Lancet Oncol. 2016;17(6):791-800.

\section{Publisher's Note}

Springer Nature remains neutral with regard to jurisdictional claims in published maps and institutional affiliations.
Ready to submit your research? Choose BMC and benefit from:

- fast, convenient online submission

- thorough peer review by experienced researchers in your field

- rapid publication on acceptance

- support for research data, including large and complex data types

- gold Open Access which fosters wider collaboration and increased citations

- maximum visibility for your research: over $100 \mathrm{M}$ website views per year

At BMC, research is always in progress.

Learn more biomedcentral.com/submissions 